\title{
THE MOMENTUM MAP FOR NONHOLONOMIC FIELD THEORIES WITH SYMMETRY
}

\author{
JORIS VANKERSCHAVER
}

\begin{abstract}
We introduce a suitable generalization of the momentum map for nonholonomic field theories and prove a covariant form of the nonholonomic momentum equation. We show that these covariant objects coincide with their counterparts in mechanics by making the transition to the Cauchy formalism.
\end{abstract}

\section{INTRODUCTION}

In this note, we study nonholonomic field theories with symmetry. Our goal is to show that the results obtained in the context of mechanical systems, such as the nonholonomic momentum map and the associated Noether theorem, have a natural counterpart in covariant field theory. We will mainly be concerned with the so-called multisymplectic approach to field theories (see [7, 8, 14] and the references therein).

In section 2 we review the multisymplectic treatment of first-order Lagrangian field theories, with special emphasis, in subsection 2.3. on the inclusion of nonholonomic constraints into this picture. The rest of the paper is then devoted to studying the action of a symmetry group: as a warming-up, we treat in section 3 the case where no constraints are present. We review the covariant Noether theorem in a way suitable for generalization to the constrained case. In section 4, we introduce constraints into the framework and we study the implications for the Noether theorem. Finally, in section 5 we break covariance to make the link with the geometric structures known from nonholonomic mechanical systems with symmetry.

\section{LAGRANGIAN FIRST-ORDER FIELD THEORIES}

2.1. Notations. Let $\pi: Y \rightarrow X$ be a fibre bundle of rank $m$, with $(n+1)$-dimensional orientable base space $X$. In addition, we will fix a volume form $\mu$ on $X$. Typically, $X$ will represent space-time and the sections of $\pi$ will be the field configurations that we wish to study. For example, in electromagnetism, $Y$ is the cotangent bundle $T^{*} X$ and the fields are 1-forms representing the electromagnetic potential. For other physically relevant examples, we refer to $[8]$.

From time to time, it will be handy to consider coordinate expressions of the objects involved: to this end, we choose a coordinate system $\left(x^{1}, \ldots, x^{n+1}\right)$ on $X$ such that $\mu$ is locally given by $\mu:=d^{n+1} x=d x^{1} \wedge \cdots \wedge d x^{n+1}$. On $Y$, we will choose a coordinate system $\left(x^{\mu}, y^{a}\right)$ adapted to the projection $\pi$ (where $a=1, \ldots, m$ ). On the first jet bundle $J^{1} \pi$ we then have the induced coordinate system $\left(x^{\mu}, y^{a}, y_{\mu}^{a}\right)$. We will denote the projection of $J^{1} \pi$ onto $Y$ by $\pi_{1,0}$, and that onto $X$ by $\pi_{1}$ (such that $\pi_{1}=\pi \circ \pi_{1,0}$ ). The bundle of $\pi_{1}$-vertical (resp. $\pi_{1,0}$-vertical) vectors on $J^{1} \pi$ will be denoted by $V \pi_{1}\left(\operatorname{resp} . V \pi_{1,0}\right)$.

The author is a Research Assistant of the Research Foundation - Flanders (FWO-Vlaanderen). 
For later use we also mention here a particular vector-valued $(n+1)$-form $S_{\mu}$ on $J^{1} \pi$, called the vertical endomorphism (see [14]). In coordinates, $S_{\mu}$ reads

$$
S_{\mu}=\left(d y^{a}-y_{\nu}^{a} d x^{\nu}\right) \wedge d^{n} x_{\mu} \otimes \frac{\partial}{\partial y_{\mu}^{a}},
$$

where $d^{n} x_{\mu}$ is a short-hand notation for $\left.\frac{\partial}{\partial x^{\mu}}\right\rfloor d^{n+1} x$.

2.2. The Cartan form. Given a regular first-order Lagrangian $L$, one can construct the associated Cartan $(n+1)$-form $\Theta_{L}$ and the multisymplectic form $\Omega_{L}=-d \Theta_{L}$. The coordinate expression of $\Theta_{L}$ is given by

$$
\Theta_{L}=\frac{\partial L}{\partial y_{\mu}^{a}}\left(d y^{a}-y_{\nu}^{a} d x^{\nu}\right) \wedge d^{n} x_{\mu}+L d^{n+1} x
$$

We will not dwell into the precise intrinsic definition of these objects any further, but instead we refer the reader to [7, 8, 14] and the references therein.

In this note, we will mainly consider the so-called De Donder-Weyl equation (see [14]): a connection $\Upsilon$ on $\pi_{1}$ with horizontal projector $\mathbf{h}$ is said to be a solution of the De DonderWeyl equation if

$$
i_{\mathbf{h}} \Omega_{L}=n \Omega_{L} .
$$

If $\mathbf{h}$ is a solution of (1) and $L$ a regular Lagrangian, then a section $\psi$ of $\pi_{1}$ is an integral section of $\mathbf{h}$ if $\psi=j^{1} \phi$ for a section $\phi$ of $\pi$ (implying that $\Upsilon$ is semi-holonomic) and, in addition, $j^{1} \phi$ satisfies the Euler-Lagrange equations:

$$
\frac{d}{d x^{\mu}}\left(\frac{\partial L}{\partial y_{\mu}^{a}}\left(j^{1} \phi\right)\right)-\frac{\partial L}{\partial y^{a}}\left(j^{1} \phi\right)=0
$$

See [2] for a proof of this statement.

2.3. Nonholonomic constraints. In this section, we will briefly show how to derive the nonholonomic equations of motion for a system with Lagrangian $L$ and a set of constraints represented by a submanifold $\mathcal{C}$. For a more detailed treatment, we refer to [2, 15].

Let $\mathcal{C}$ be a $k$-codimensional submanifold of $J^{1} \pi$, with $\pi_{1,0}(\mathcal{C})=Y$ and such that $\left(\pi_{1,0}\right)_{\mid \mathcal{C}}$ : $\mathcal{C} \rightarrow Y$ is a subbundle of $\pi_{1,0}$. The submanifold $\mathcal{C}$ will represent some external (nonholonomic) constraints imposed on the system. Assume that $\mathcal{C}$ is locally given by the vanishing of $k$ independent functions $\varphi^{\alpha}$ and consider the subset $F$ of $\wedge^{n+1}\left(T^{*} J^{1} \pi\right)$ spanned by $\Phi^{\alpha}=S_{\mu}^{*}\left(d \varphi^{\alpha}\right)$, where $S_{\mu}$ is the vertical endomorphism on $J^{1} \pi$. In coordinates, we have

$$
\Phi^{\alpha}=\frac{\partial \varphi^{\alpha}}{\partial y_{\mu}^{a}}\left(d y^{a}-y_{\nu}^{a} d x^{\nu}\right) \wedge d^{n} x_{\mu} .
$$

The $(n+1)$-forms $\Phi^{\alpha}$ are linearly independent because of the initial assumption that $\left(\pi_{1,0}\right)_{\mid \mathcal{C}}$ is a subbundle of $\pi_{1,0}$. Hence, $F$ is a subbundle of $\wedge^{n+1}\left(T^{*} J^{1} \pi\right)$.

In the presence of nonholonomic constraints, the field equations become

$$
\frac{d}{d x^{\mu}}\left(\frac{\partial L}{\partial y_{\mu}^{a}}\left(j^{1} \phi\right)\right)-\frac{\partial L}{\partial y^{a}}=\lambda_{\alpha \mu} \frac{\partial \varphi^{\alpha}}{\partial y_{\mu}^{a}},
$$


together with the constraint that $j^{1} \phi \in \mathcal{C}$ (see [2]). Accordingly, the unconstrained De Donder-Weyl equations are replaced by the following conditions along $\mathcal{C}$ :

$$
i_{\mathbf{h}} \Omega_{L}-n \Omega_{L} \in \mathcal{I}(F) \text { and } \operatorname{Im} \mathbf{h} \subset T \mathcal{C},
$$

where $\mathcal{I}(F)$ is the ideal generated by $F$. The terms on the right-hand side of (3) and (4) represent the constraint forces that keep the section $j^{1} \phi$ constrained to $\mathcal{C}$. The unknown multipliers $\lambda_{\alpha \mu}$ should be determined from the condition that $j^{1} \phi \in \mathcal{C}$.

Remark 2.1. In general, the constraints represented by the submanifold $\mathcal{C}$ are nonlinear. Linear constraints can be treated as a special case of this formalism by considering a distribution $D$ on $Y$ and taking $\mathcal{C}$ to be

$$
\mathcal{C}=\left\{j_{x}^{1} \phi \in J^{1} \pi: \operatorname{Im} T_{x} \phi \subset D_{\phi(x)}\right\} .
$$

If $D$ is annihilated by the $k$ one-forms $A_{a}^{\alpha} d y^{a}+B_{\mu}^{\alpha} d x^{\mu}$, then $\mathcal{C}$ is given by the vanishing of the $k n$ functions $\varphi_{\mu}^{\alpha}=A_{a}^{\alpha} y_{\mu}^{a}+B_{\mu}^{\alpha}$. Whenever $D$ is integrable, these constraint functions can be written as total derivatives with respect to $x^{\mu}$ of functions on $Y$, in which case the constraints can reasonably be said to be holonomic. This case is treated in far greater detail in [12.

2.4. Connections on $\pi_{1}$. In this section, we will prove a number of straightforward properties of connections on $\pi_{1}$ that will be useful later on. The reader is referred to [14] for a more comprehensive treatment.

We recall that a connection $\Upsilon$ on $\pi_{1}$ is said to be semi-holonomic if the associated horizontal projector $\mathbf{h}$ satisfies $i_{\mathbf{h}} \theta=0$ for each contact one-form $\theta$. In coordinates, if

$$
\mathbf{h}=d x^{\mu} \otimes\left(\frac{\partial}{\partial x^{\mu}}+\Gamma_{\mu}^{a} \frac{\partial}{\partial y^{a}}+\Gamma_{\mu \nu}^{a} \frac{\partial}{\partial y_{\nu}^{a}}\right),
$$

semi-holonomicity implies that $\Gamma_{\mu}^{a}=y_{\mu}^{a}$. This implies that any integral section of $\mathbf{h}$ is automatically the prolongation of a section of $\pi$.

Lemma 2.2. For each semi-holonomic connection $\Upsilon$ with horizontal projector $\mathbf{h}$, the following holds:

$$
i_{\mathbf{h}} \Theta_{L}=n \Theta_{L}+L \mu
$$

Proof: We give the proof in coordinates. For any connection $\mathbf{h}$, we have

$$
i_{\mathbf{h}} d^{n+1} x=(n+1) d^{n+1} x \quad \text { and } \quad i_{\mathbf{h}} d^{n} x_{\mu}=n d^{n} x_{\mu} .
$$

Therefore,

$$
i_{\mathbf{h}} \Theta_{L}=\frac{\partial L}{\partial y_{\nu}^{a}} i_{\mathbf{h}} \theta^{a} \wedge d^{n} x_{\nu}+n \frac{\partial L}{\partial y_{\nu}^{a}} \theta^{a} \wedge d^{n} x_{\nu}+(n+1) L d^{n+1} x,
$$

where we have introduced the contact forms $\theta^{a}=d y^{a}-y_{\mu}^{a} d x^{\mu}$. If $\mathbf{h}$ is semi-holonomic, the first term on the right-hand side is zero and we obtain the desired expression.

This lemma can be seen as the jet-bundle analogue of the well-known fact in Lagrangian mechanics that $i_{X} \theta_{L}=\Delta(L)$ for any second-order vector field $X$, where $\theta_{L}$ is the Cartan one-form corresponding to $L$, and $\Delta$ the Liouville vector field.

Lemma 2.3. Let $X$ be a vertical vector field on $Y$ and $X^{(1)}$ its prolongation to $J^{1} \pi$. If $\Upsilon$ is a semi-holonomic connection on $\pi_{1}$ with horizontal projector $\mathbf{h}$, then the Frölicher-Nijenhuis bracket $\left[X^{(1)}, \mathbf{h}\right]$ is a vector-valued one-form taking values in $V \pi_{1,0}$. 
Proof: If $X=X^{a} \frac{\partial}{\partial y^{a}}$, then

$$
X^{(1)}=X^{a} \frac{\partial}{\partial y^{a}}+\left(\frac{\partial X^{a}}{\partial x^{\mu}}+\frac{\partial X^{a}}{\partial y^{b}} y_{\mu}^{b}\right) \frac{\partial}{\partial y_{\mu}^{a}} .
$$

(see e.g. [14]) For the bracket, we have that $\left[X^{(1)}, \mathbf{h}\right]=\mathscr{L}_{X^{(1)}} \mathbf{h}$ and a straightforward calculation then shows that this is a semi-basic vector-valued one-form taking values in $V \pi_{1}$. We now focus on the coefficient of $d x^{\mu} \otimes \frac{\partial}{\partial y^{a}}$, which is just

$$
X^{(1)}\left(\Gamma_{\mu}^{a}\right)-\left(\frac{\partial X^{a}}{\partial x^{\mu}}+\Gamma_{\mu}^{b} \frac{\partial X^{a}}{\partial y^{b}}\right) .
$$

This coefficient is easily seen to vanish when $\Gamma_{\mu}^{a}=y_{\mu}^{a}$, i.e. when $\mathbf{h}$ is semi-holonomic, which completes the proof.

As a corollary, we note that this lemma implies that the contraction of $\left[X^{(1)}, \mathbf{h}\right]$ with a semibasic form (in particular with $\Theta_{L}$ ) vanishes.

\section{Symmetry in the ABSEnCE of nOnholonomic CONSTRAints}

Let $G$ be a Lie group acting on $Y$ by bundle automorphisms $\Phi_{g}$ over the identity in $X$. The assumption that $G$ acts vertically is probably superfluous, but for the sake of clarity we will assume it nevertheless.

The Lie group $G$ acts on $J^{1} \pi$ by prolonged bundle automorphisms, i.e. $j^{1} \Phi_{g}\left(j_{x}^{1} \phi\right)=j_{x}^{1}\left(\Phi_{g} \circ \phi\right)$. Now, let $L \in C^{\infty}\left(J^{1} \pi\right)$ be a $G$-invariant Lagrangian. The action of $G$ on $J^{1} \pi$ is called Lagrangian if, for each $\xi \in \mathfrak{g}$, there exists an $n$-form $J_{\xi}$ (depending linearly on $\xi$ ) such that $i_{\xi_{J^{1} \pi}} \Omega_{L}=d J_{\xi}$, where $\xi_{J^{1} \pi}$ denotes the infinitesimal generator corresponding to $\xi$. In this case, the map $J: J^{1} \pi \rightarrow \wedge^{n}\left(T^{*} J^{1} \pi\right) \otimes \mathfrak{g}^{*}$ defined by $\langle J, \xi\rangle:=J_{\xi}$ is called the covariant momentum map for the action $\Phi$.

In general, we can also consider actions of $G$ on $J^{1} \pi$ that are not prolonged actions of an action on $Y$, but in this note we will nevertheless restrict ourselves to this special case. It is easy to see that Lagrangian actions satisfy $\mathscr{L}_{\xi_{J} \pi_{\pi}} \Omega_{L}=0$; for prolonged actions we have in addition that $\mathscr{L}_{\xi_{J^{1} \pi}} \Theta_{L}=0$ (see lemma 3.1).

If $G$ acts on $J^{1} \pi$ by prolonged bundle automorphisms, then for each $\xi \in \mathfrak{g}$ the infinitesimal generator $\xi_{J^{1} \pi}$ on $J^{1} \pi$ is the prolongation of the infinitesimal generator $\xi_{Y}$ on $Y$. From now on, we will denote $\xi_{J^{1} \pi}$ by $\xi^{(1)}$.

Lemma 3.1. The Cartan $(n+1)$-form $\Theta_{L}$ is invariant with respect to the action of $G$ lifted to $J^{1} \pi$ :

$$
\mathscr{L}_{\xi^{(1)}} \Theta_{L}=0
$$

Proof: See [8, p. 45].

For a prolonged action, there always exists a covariant momentum map which is explicitly given by

$$
J_{\xi}=i_{\xi^{(1)}} \Theta_{L}
$$

(see [8, p. 45]). The importance of the covariant momentum map lies in the covariant Noether theorem, first proved in [8]. 
Proposition 3.2 (Covariant Noether theorem). Let $\Upsilon$ be a connection on $\pi_{1}$ such that the associated horizontal projector $\mathbf{h}$ is a solution of the unconstrained De Donder-Weyl equation (1). For every $\xi \in \mathfrak{g}$, the momentum map $J_{\xi}$ is constant on integral sections of $\mathbf{h}$ :

$$
d_{\mathbf{h}} J_{\xi}=0 \text {. }
$$

Proof: In this proof, as well as in the remainder of this note, we make frequent use of some elementary properties of the Frölicher-Nijenhuis bracket. For the sake of completeness, we have summarized these properties in the appendix.

We have

$$
\begin{aligned}
d_{\mathbf{h}} J_{\xi} & =d_{\mathbf{h}} i_{\xi^{(1)}} \Theta_{L} \\
& =\left(i_{\mathbf{h}} d-d i_{\mathbf{h}}\right) i_{\xi^{(1)}} \Theta_{L} \\
& =i_{\mathbf{h}} \mathscr{L}_{\xi^{(1)}} \Theta_{L}-i_{\mathbf{h}} i_{\xi^{(1)}} d \Theta_{L}-d i_{\mathbf{h}} i_{\xi^{(1)}} \Theta_{L} .
\end{aligned}
$$

In the last expression, the first term vanishes because of lemma 3.1. The second term can be rewritten by using the field equations (note that $\mathbf{h}\left(\xi^{(1)}\right)=0$ as $\xi^{(1)}$ is $\pi_{1}$-vertical):

$$
i_{\mathbf{h}} i_{\xi^{(1)}} d \Theta_{L}=i_{\xi^{(1)}} i_{\mathbf{h}} d \Theta_{L}=-n i_{\xi^{(1)}} \Omega_{L},
$$

whereas for the last term we have, using lemma 2.3 .

$$
\begin{aligned}
d i_{\mathbf{h}} i_{\xi^{(1)}} \Theta_{L} & =d i_{\xi^{(1)}} i_{\mathbf{h}} \Theta_{L} \\
& =d i_{\xi^{(1)}}\left(n \Theta_{L}+L \mu\right) .
\end{aligned}
$$

Now, $i_{\xi^{(1)}}(L \mu)=0$ and so we obtain

$$
d_{\mathbf{h}} J_{\xi}=n i_{\xi^{(1)}} \Omega_{L}-n d i_{\xi^{(1)}} \Theta_{L}=-n \mathscr{L}_{\xi^{(1)}} \Theta_{L}=0,
$$

again due to the invariance of $\Theta_{L}$.

Remark 3.3. In [8, p. 45], the authors prove a slightly different Noether theorem. They show that, if $\phi$ is a solution of the field equations, then $d\left(j^{1} \phi\right)^{*} J_{\xi}=0$. It is not hard to prove that, for any $k$-form $\alpha$ on $J^{1} \pi,\left(j^{1} \phi\right)^{*} d_{\mathbf{h}} \alpha=d\left(j^{1} \phi\right)^{*} \alpha$ if and only if $j^{1} \phi$ is an integral section of $\mathbf{h}$. Proposition 3.2 therefore implies that $d\left(j^{1} \phi\right)^{*} J_{\xi}=0$. The proof of the Noether theorem in [8] is more straightforward; our proof has the advantage that it will be easily extendible to the case where nonholonomic constraints are present.

\section{The COnstrained MOMEntum MaP}

In this section, we study the case of a constrained field theory, with regular Lagrangian $L$ and constraint submanifold $\mathcal{C}$ satisfying the assumptions of section 2.3 , The constrained De Donder-Weyl equations are then given by (4).

Suppose now that in addition to these nonholonomic constraints, there is also a symmetry group $G$ acting on $J^{1} \pi$ by prolonged bundle automorphisms, such that both the Lagrangian $L$ and the constraint manifold $\mathcal{C}$ are $G$-invariant, i.e.

$$
L \circ j^{1} \Phi_{g}=L \quad \text { as well as } \quad j^{1} \Phi_{g}(\mathcal{C}) \subset \mathcal{C}
$$

for all $g \in G$. In general, as in the case of nonholonomic mechanics (see [1, 4, 5]), it will no longer be true that these symmetries give rise to conserved quantities; the precise link will be made clear by the nonholonomic momentum equation or constrained Noether theorem (theorem 4.1). Our treatment extends the one in [5]; we refer to that paper, as well as to 
[1, 4] and the references therein, for more information about the nonholonomic momentum equation in mechanics.

We first introduce the following distribution:

$$
\mathcal{E}(\gamma)=\left\{v \in T_{\gamma} J^{1} \pi: i_{v}\left(S_{\mu}^{*} d \varphi_{\alpha}\right)=0 \text { for each } \alpha=1, \ldots, k\right\} \quad \text { where } \gamma \in \mathcal{C} .
$$

For a given $\gamma \in \mathcal{C}$ we consider all elements $\xi$ of the Lie algebra $\mathfrak{g}$ such that $\xi^{(1)}(\gamma) \in \mathcal{E}(\gamma)$. The set of all such $\xi$ we denote by $\mathfrak{g}^{\gamma}$. We take $\mathfrak{g}^{\mathcal{E}}$ to be the disjoint union of all these spaces $\mathfrak{g}^{\gamma}$ and we assume that $\mathfrak{g}^{\mathcal{E}}$ can be given the structure of a bundle over $\mathcal{C}$.

With these elements in mind, we define the constrained momentum map as a map $J^{\text {n.h. }}: \mathcal{C} \rightarrow$ $\wedge^{n}\left(J^{1} \pi\right) \otimes g^{\mathcal{E}}$, constructed as follows. With every section $\bar{\xi}$ of $\mathfrak{g}^{\mathcal{E}}$, one may associate a vector field $\tilde{\xi}$ on $J^{1} \pi$ by putting $\tilde{\xi}(\gamma)=(\bar{\xi}(\gamma))_{J^{1} \pi}(\gamma)$. Remark that $\tilde{\xi}$ is a section of $\mathcal{E}$. We then define $J_{\bar{\xi}}^{\text {n.h. }}$ along $\mathcal{C}$ as

$$
J_{\bar{\xi}}^{\text {n.h. }}=i_{\tilde{\xi}} \Theta_{L} .
$$

The importance of the nonholonomic momentum map lies in the nonholonomic momentum equation:

Theorem 4.1 (Nonholonomic momentum equation). Let $\Upsilon$ be a connection on $\pi_{1}$ such that the associated horizontal projector $\mathbf{h}$ is a solution of the constrained De Donder-Weyl equation. Assume furthermore that $G$ is a Lie group acting on $J^{1} \pi$ in the way described above. Then the nonholonomic momentum map satisfies the following equation:

$$
d_{\mathbf{h}} J_{\bar{\xi}}^{\text {n.h. }}=\mathscr{L}_{\tilde{\xi}}(L \mu) \quad \text { along } \mathcal{C} .
$$

Proof: Equation (5) from the proof of proposition 3.2 can be used without modification:

$$
\begin{aligned}
d_{\mathbf{h}} J_{\bar{\xi}}^{\text {n.h. }} & =i_{\mathbf{h}} \mathscr{L}_{\tilde{\xi}} \Theta_{L}-i_{\mathbf{h}} i_{\tilde{\xi}} d \Theta_{L}-d i_{\mathbf{h}} i_{\tilde{\xi}} \Theta_{L} \\
& =i_{\mathbf{h}} \mathscr{L}_{\tilde{\xi}} \Theta_{L}+i_{\tilde{\xi}}\left(n \Omega_{L}+\zeta\right)-n \mathscr{L}_{\tilde{\xi}} \Theta_{L}+n i_{\tilde{\xi}} d \Theta_{L} .
\end{aligned}
$$

In this expression, we have substituted the constrained De Donder-Weyl equation: $\zeta$ is an element of $\mathcal{I}(F)$. As $\zeta$ can be written as $\zeta=\lambda_{\alpha \mu} d x^{\mu} \wedge f^{\alpha}$ (see [15]), with $f^{\alpha}$ taking values in the bundle $F$, we may conclude that $i_{\tilde{\xi}} \zeta=0$. Therefore, we end up with

$$
\begin{aligned}
d_{\mathbf{h}} J_{\tilde{\xi}}^{\text {n.h. }} & =i_{\mathbf{h}} \mathscr{L}_{\tilde{\xi}} \Theta_{L}-n \mathscr{L}_{\tilde{\xi}} \Theta_{L} \\
& =\mathscr{L}_{\tilde{\xi}} i_{\mathbf{h}} \Theta_{L}-i_{[\tilde{\xi}, \mathbf{h}]} \Theta_{L}-n \mathscr{L}_{\tilde{\xi}} \Theta_{L} \\
& =\mathscr{L}_{\tilde{\xi}}(L \mu),
\end{aligned}
$$

where we have used the remark following lemma 2.3 to conclude that $i_{[\tilde{\xi}, \mathbf{h}]} \Theta_{L}=0$.

We finish by noting that in the case where $\tilde{\xi}$ can be written as $\xi^{(1)}$ (for example, when $\bar{\xi}$ is a constant section), we may conclude from the $G$-invariance of $L$ that $d_{\mathbf{h}} J_{\tilde{\xi}}^{\text {n.h. }}=0$. In general, though, this will not be the case.

\section{The Cauchy formalism}

Up until now, all of our results have been derived in a purely covariant setting where all of the coordinates on the base space $X$ are treated on an equal footing. In particular, there is no distinguised time coordinate. We will now assume that the Euler-Lagrange equations associated to the Lagrangian $L$ describe an (hyperbolic) initial-value problem. In this case, 
it is meaningful to single out a global direction of time and break covariance by making the transition to the space of Cauchy data. We can then rephrase the field equations accordingly as a time-dependent mechanical system on an infinite-dimensional configuration space (see [6, 9, 13]).

This is done by fixing a particular diffeomorphism $\Psi: \mathbb{R} \times M \rightarrow X$, where $M$ is an $n$ dimensional manifold (and where we tacitly assume that the topology of $X$ is such that $\Psi$ can indeed be globally defined), thus singling out a "splitting" of $X$ into space and time. To avoid the technical matters arising when considering the behaviour of the field "at infinity", we assume that $M$ is compact. We define the space $\tilde{X}$ to consist of all embeddings $\tau$ of $M$ into $X$ such that there exists a $t \in \mathbb{R}$ for which $\tau=\Psi(t, \cdot)$. Hence, there is a one-to-one correspondence between $\mathbb{R}$ and $\tilde{X}$. This correspondence, or the existence of the diffeomorphism $\Psi$, induces a distinguished vector field $\mathbf{T}$ on $X$, defined at $x \in X$, by

$$
\mathbf{T}(x)=\left.\frac{d}{d s} \Psi(s, u)\right|_{s=t}, \quad \text { where } x=\Psi(t, u) .
$$

For the sake of convenience, we will assume that $M$ is equipped with a volume form $\mu_{M}$ such that $\mu:=d t \wedge \mu_{M}$ is a volume form for $X$, where $t$ is a global coordinate labelling $\tilde{X}$.

We define the space of Cauchy data (denoted by $\tilde{Z}$ ) as the space of embeddings $\kappa: M \hookrightarrow J^{1} \pi$ for which there exists a section $\phi$ of $\pi$ and an element $\tau$ of $\tilde{X}$ such that $\kappa=j^{1} \phi \circ \tau$. For more information on this space (which can be given the structure of a smooth manifold in some suitable sense), we refer the reader to [3, 9, 11, 13]. There exists a convenient way of viewing the tangent bundle of $\tilde{Z}$ : a tangent vector $v \in T_{\kappa} \tilde{Z}$ can be seen as a section of $\Gamma\left(\kappa^{*} T J^{1} \pi\right)$ (a vector field along $\kappa$ ). There exist similar interpretations of $T \tilde{X}$ and $T \tilde{Y}$.

A vector field $V$ on $J^{1} \pi$ induces a vector field $\tilde{V}$ on $\tilde{Z}$ by composition: $\tilde{V}(\kappa)=V \circ \kappa$. Similarly, an $(n+k)$-form $\alpha$ on $J^{1} \pi$ induces a $k$-form $\tilde{\alpha}$ on $\tilde{Z}$ by integration:

$$
\tilde{\alpha}(\kappa)\left(\tilde{V}_{1}, \ldots, \tilde{V}_{k}\right)=\int_{M} \kappa^{*} i_{\tilde{V}_{1} \wedge \cdots \wedge \tilde{V}_{k}} \alpha .
$$

By use of this correspondence, the multisymplectic form $\Omega_{L}$ and the volume form $\mu$ induce respectively a two-form $\tilde{\Omega}_{L}$ and a one-form $\tilde{\mu}$ on $\tilde{Z}$, whereas the Lagrangian $L$ can be seen to induce a function on $\tilde{Z}$ :

$$
\tilde{L}(\kappa)=\int_{M} \kappa^{*} i_{\mathbf{T}}(L \mu)
$$

Strictly speaking, on the right-hand side of this expression one should replace $\mathbf{T}$ by an arbitrary vector field $V$ on $J^{1} \pi$ projecting down to $\mathbf{T}$, but since $L \mu$ is semi-basic, the contraction does not depend on $V$ but only on $\mathbf{T}$.

Remark 5.1. It has been shown that the covariant field equations induce a dynamical system $\Gamma$ on $\tilde{Z}$ whose determining equations are formally identical to those of a time-dependent mechanical system with an infinite-dimensional configuration space (see [9, 13]):

$$
i_{\Gamma} \tilde{\Omega}_{L}=0 \quad \text { and } \quad i_{\Gamma} \tilde{\mu}=1 .
$$

In [15, we showed that in the case of nonholonomic field theory, the induced dynamical system on $\tilde{Z}$ is determined by

$$
\left.i_{\Gamma} \tilde{\Omega}_{L}\right|_{\tilde{\mathcal{C}}} \in \tilde{F} \quad \text { and } \quad \Gamma \in T \tilde{\mathcal{C}}
$$


where $\tilde{F}$ is a codistribution induced by $F$ and $\tilde{\mathcal{C}}$ is the subset of $\tilde{Z}$ induced by $\mathcal{C}$ and defined as

$$
\tilde{\mathcal{C}}=\{\kappa \in \tilde{Z}: \operatorname{Im} \kappa \subset \mathcal{C}\}
$$

In both the constrained and the unconstrained case, a connection $\Upsilon$ solving the covariant field equations induces a vector field $\Gamma$ on $\tilde{Z}$ which is a solution of the corresponding dynamical system on $\tilde{Z}$. In the unconstrained case, this dynamical system is given by (7), whereas in the constrained case the equations of motion are given by (8). The precise relation between $\mathbf{h}$ and $\Gamma$ is

$$
\Gamma(\kappa)=\mathbf{h}\left(T j^{1} \phi(\mathbf{T})\right) \circ \kappa,
$$

where we have decomposed $\kappa$ as $\kappa=j^{1} \phi \circ \tau$. With some abuse of notation, we will also write $\Gamma=\mathbf{h}(\mathbf{T})$.

In the next sections, we will exhibit the structures on $\tilde{Z}$ induced by the (nonholonomic) momentum map and we will show how the covariant momentum equation give rises to a momentum equation on $\tilde{Z}$ which is formally identical to the one encountered in nonholonomic mechanics (see for example [4, [5]).

By (6), the component $J_{\xi}: J^{1} \pi \rightarrow \wedge^{n}\left(J^{1} \pi\right)$ of the covariant momentum map induces a map $\tilde{J}_{\xi} \in C^{\infty}(\tilde{Z})$ on the space of Cauchy data:

$$
\tilde{J}_{\xi}(\kappa)=\int_{M} \kappa^{*} J_{\xi}
$$

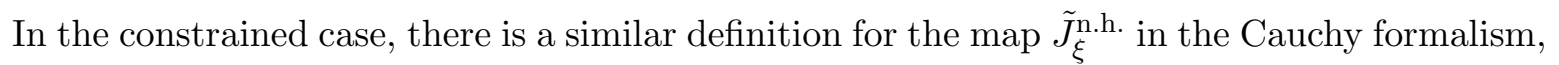
induced by the component $J_{\xi}^{\text {n.h. }}$ of the constrained momentum map. Note that $J_{\xi}^{\text {n.h. is defined }}$ along $\mathcal{C}$.

5.1. The unconstrained case. We now turn to proving the analogue of Noether's theorem in the Cauchy framework. There are essentially two ways in which one could approach this problem: either by directly defining the action of $G$ on $\tilde{Z}$ and using the standard techniques known from mechanics, or by showing that the covariant Noether theorem leads in a straightforward way to the Noether theorem on the space of Cauchy data. We choose to follow the second approach, as it allows us to postpone to the very end all of the technical matters associated with the calculus on infinite-dimensional manifolds.

Proposition 5.2. Let $\Upsilon$ be a connection in $\pi_{1}$ such that the associated horizontal projector $\mathbf{h}$ is a solution of the De Donder-Weyl equation (1). Let $\tilde{J}$ be the momentum map associated to the covariant momentum map J. Then Noether's theorem holds: $\Gamma\left(\tilde{J}_{\xi}\right)=0$ for all $\xi \in \mathfrak{g}$, where $\Gamma$ is a solution to the equations of motion (7) in the Cauchy formalism.

Proof: We will use the following characterisation of the exterior derivative $d \tilde{J}_{\xi}$ in terms of $d J_{\xi}:$

$$
\left\langle\tilde{V}, d \tilde{J}_{\xi}\right\rangle(\kappa)=\int_{M} \kappa^{*}\left(i_{\tilde{V}} d J_{\xi}\right)
$$

for an arbitrary vector field $\tilde{V}$ on $\tilde{Z}$. For a proof, we refer to [13, prop. 3.3.9] or to the expressions used in [9, lemma 5.1].

The embedding $\kappa: M \hookrightarrow J^{1} \pi$ can be written as $\kappa=j^{1} \phi \circ \tau$. Without loss of generality, we may take $\phi$ to be a solution of the field equations. This lies at the heart of the Cauchy analysis: $\kappa$ specifies the values of the fields and their derivatives on a hypersurface and due 
to the (supposed) hyperbolicity of the equations of motion, the subsequent evolution is then fixed (and given by $j^{1} \phi$ ). Formally, let $t \mapsto c(t)$ be an integral curve of $\Gamma$ such that $c(0)=\kappa$. Then $j_{x}^{1} \phi=[c(t)](u)$, where $x=\Phi(t, u)$.

We then have, noting that $\mathbf{h}(\mathbf{T})=T j^{1} \phi(\mathbf{T})$,

$$
\left\langle\Gamma, d \tilde{J}_{\xi}\right\rangle(\kappa)=\int_{M} \kappa^{*}\left(i_{\Gamma} d J_{\xi}\right)=\int_{M} \tau^{*}\left(j^{1} \phi\right)^{*}\left(i_{\mathbf{h}(\mathbf{T})} d J\right)=\int_{M} \tau^{*} i_{\mathbf{T}}\left(\left(j^{1} \phi\right)^{*} d J\right) .
$$

As we pointed out in the remark following proposition 3.2 , one can check that $\left(j^{1} \phi\right)^{*} d_{\mathbf{h}} \alpha=$ $d\left(j^{1} \phi\right)^{*} \alpha$ if and only if $j^{1} \phi$ is an integral section of $\mathbf{h}$. We conclude that

$$
\left\langle\Gamma, d \tilde{J}_{\xi}\right\rangle(\kappa)=\int_{M} \tau^{*} i_{\mathbf{T}}\left(\left(j^{1} \phi\right)^{*} d_{\mathbf{h}} J_{\xi}\right) .
$$

As the $\xi$-component $J_{\xi}$ of the covariant momentum map satisfies Noether's theorem, i.e. $d_{\mathbf{h}} J_{\xi}=0$, we have that $\Gamma\left(\tilde{J}_{\xi}\right)=0$. This establishes the theorem of Noether in the Cauchy framework.

5.2. The constrained case. Quite surprisingly, much of the material developed in the preceding section carries over quite naturally to the constrained case. In particular, for the nonholonomic momentum map, equation (10) still holds:

$$
\left\langle\Gamma, d \tilde{J}_{\bar{\xi}}^{\text {n.h. }}\right\rangle(\kappa)=\int_{M} \tau^{*} i_{\mathbf{T}}\left(\left(j^{1} \phi\right)^{*} d_{\mathbf{h}} J_{\bar{\xi}}^{\text {n.h. }}\right), \quad \text { for } \kappa \in \mathcal{C},
$$

where we attribute a similar meaning to all terms involved: $\mathbf{h}$ is a solution of the constrained De Donder-Weyl equation, $j^{1} \phi$ is an integral section of the corresponding connection and $\Gamma=\mathbf{h}(\mathbf{T})$. Note that $\Gamma$ is now a solution of (8).

Now, if $J_{\bar{\xi}}^{\text {n.h. }}$ satisfies the nonholonomic momentum equation, then

$$
\left\langle\Gamma, d \tilde{J}_{\bar{\xi}}^{\text {n.h. }}\right\rangle(\kappa)=\int_{M} \tau^{*} i_{\mathbf{T}}\left(\left(j^{1} \phi\right)^{*} \mathscr{L}_{\bar{\xi}}(L \mu)\right)
$$

In the following proposition, we further elaborate the right-hand side. We recall that the vector field $\tilde{\xi}$ on $J^{1} \pi$ naturally induces a vector field $\hat{\xi}$ on $\tilde{Z}$ by putting $\hat{\xi}(\kappa)=\tilde{\xi} \circ \kappa$.

Proposition 5.3. Let $\Upsilon$ be a connection on $\pi_{1}$ such that along the constraint submanifold $\mathcal{C}$ the associated horizontal projector $\mathbf{h}$ satisfies the constrained De Donder-Weyl equation. Assume a Lie group $G$ acts in the way described above and let $\tilde{J}^{\text {n.h. }}$ be the momentum map associated to the covariant momentum map $J^{\text {n.h. }}$. Then $\tilde{J}^{\text {n.h. }}$ satisfies the nonholonomic momentum equation: for all $\bar{\xi} \in \mathfrak{g}^{\mathcal{E}}$,

$$
\Gamma\left(\tilde{J}_{\bar{\xi}}^{\text {n.h. }}\right)=\hat{\xi}(\tilde{L}) \quad \text { along } \mathcal{C} .
$$

Proof: We rewrite the right-hand side of (11) by performing exactly the opposite manipulations as we did to obtain eq. (10). This leads to

$$
\left\langle\Gamma, d \tilde{J}_{\bar{\xi}}^{\text {n.h. }}\right\rangle(\kappa)=\int_{M} \kappa^{*} i_{\mathbf{h}(\mathbf{T})} \mathscr{L}_{\tilde{\xi}}(L \mu)=\int_{M} \kappa^{*} \mathscr{L}_{\tilde{\xi}}\left(i_{\mathbf{h}(\mathbf{T})}(L \mu)\right)+\int_{M} \kappa^{*} i_{[\mathbf{h}(\mathbf{T}), \tilde{\xi}]}(L \mu) .
$$

The last term vanishes as $L \mu$ is semi-basic and $[\mathbf{h}(\mathbf{T}), \tilde{\xi}]$ is $\pi_{1}$-vertical $\left(\tilde{\xi}\right.$ is $\pi_{1}$-vertical). By lemma 3.3 .9 of [13], we see that the first term on the right-hand side equals

$$
\int_{M} \kappa^{*} \mathscr{L}_{\tilde{\xi}}\left(i_{\mathbf{h}(\mathbf{T})}(L \mu)\right)=\mathscr{L}_{\hat{\xi}}(\tilde{L})
$$


and this proves the momentum equation in the Cauchy formalism.

\section{ACKNOWLEDGEMENTS}

Financial support of the Research Foundation-Flanders (FWO-Vlaanderen) is gratefully acknowledged. I would also like to thank Frans Cantrijn for useful discussions and a critical reading of this manuscript, as well as Manuel de León and David Martín de Diego for

many fruitful discussions and their kind hospitality during several research visits to the CSIC (Madrid).

\section{APPENDiX: ELEMENTARY PROPERTIES OF THE FrÖLICHER-NiJENHUIS BRACKET}

In this section, we review some properties of the Frölicher-Nijenhuis bracket and the various derivations associated to vector-valued forms on a manifold. For a detailed treatment of the Frölicher-Nijenhuis bracket, we refer the reader to [10, 14].

Let $M$ be a manifold. A vector-valued one-form $\mathbf{h}$ is a section of $T M \otimes T^{*} M$. Associated to $\mathbf{h}$ is a derivation $i_{\mathbf{h}}$ (of type $i_{*}$ and degree 0 ), defined by

$$
\left(i_{\mathbf{h}} \alpha\right)\left(v_{0}, \ldots, v_{k}\right)=\sum_{i=0}^{k}(-1)^{i} \alpha\left(\mathbf{h}\left(v_{i}\right), v_{0}, \ldots, \widehat{v}_{i}, \ldots, v_{k}\right) \quad \text { for } \alpha \in \Omega^{k+1}(M) .
$$

We then define $d_{\mathbf{h}}$ as $d_{\mathbf{h}}=i_{\mathbf{h}} \circ d-d \circ i_{\mathbf{h}}$; this is a derivation of type $d_{*}$ and degree 1 .

Vector-valued forms of higher degree are defined accordingly as sections of the tensor product $T M \otimes \wedge^{k}\left(T^{*} M\right)$. A vector-valued $k$-form $R$ can easily be seen to give rise to a derivation $i_{R}$ of degree $k-1$ (by virtue of a generalization of eq. 12) as well as a derivation $d_{R}$ of degree $k$. A vector-valued form of degree zero is simply a vector field, and the associated derivations are in this case the contraction $i_{X}$ and the Lie derivative $\mathscr{L}_{X}$.

The Frölicher-Nijenhuis bracket of a vector-valued $r$-form $R$ and a vector-valued $s$-form $S$ is then defined as the unique vector-valued $(r+s)$-form $[R, S]$ for which

$$
d_{R} \circ d_{S}-(-1)^{r s} d_{S} \circ d_{R}=d_{[R, S]} .
$$

We have deliberately been vague about the nature of this bracket: most of the time we will only need the bracket of a vector field $X$ with a vector-valued one-form $\mathbf{h}$ (which will be the horizontal projector of a connection). In this case, it is not hard to prove that

$$
[X, \mathbf{h}]=\mathscr{L}_{X} \mathbf{h} .
$$

The following lemma collects the properties of the Frölicher-Nijenhuis bracket that we will be needing in the body of the text. They can be suitably generalized and form part of a well-investigated calculus, for which we refer to [10.

Lemma 5.4. Let $X$ be a vector field on $M$ and $\mathbf{h}$ a vector-valued one-form. Then, for any $k$-form $\alpha$ on $M$, the following holds:

(1) $i_{X} i_{\mathbf{h}} \alpha=i_{\mathbf{h}} i_{X} \alpha+i_{\mathbf{h}(X)} \alpha$;

(2) $i_{\mathbf{h}} \mathscr{L}_{X} \alpha=\mathscr{L}_{X} i_{\mathbf{h}} \alpha-i_{[X, \mathbf{h}]} \alpha$. 
Proof: Let $\alpha$ be a 2-form (the case of a $k$-form $\alpha$ is completely similar) and $Y$ a vector field on $M$. Then

$$
\begin{aligned}
\left(i_{X} i_{\mathbf{h}} \alpha\right)(Y) & =\alpha(\mathbf{h}(X), Y)-\alpha(\mathbf{h}(Y), X) \\
& =\left(i_{\mathbf{h}(X)} \alpha\right)(Y)+\left(i_{\mathbf{h}} i_{X} \alpha\right)(Y),
\end{aligned}
$$

which confirms the first property.

The second property (a special case of lemma 8.6 in [10]) can be proved directly by noting that a derivation is completely determined by its action on functions and one-forms. For a function $f$ both sides of the relation (2) vanish and for a one-form $\alpha$ we have for the left-hand side

$$
\begin{aligned}
\left(i_{\mathbf{h}} \mathscr{L}_{X} \alpha\right)(Y) & =\left(\mathscr{L}_{X} \alpha\right)(\mathbf{h}(Y))=\mathscr{L}_{X}(\alpha(\mathbf{h}(Y)))-\alpha([X, \mathbf{h}(Y)]) \\
& =\mathscr{L}_{X}(\alpha(\mathbf{h}(Y)))-\alpha\left(\left(\mathscr{L}_{X} \mathbf{h}\right)(Y)\right)-\alpha(\mathbf{h}([X, Y])) .
\end{aligned}
$$

Taking together the first and third term, we obtain $\mathscr{L}_{X}\left(i_{\mathbf{h}} \alpha\right)(Y)$, whereas the second term is just $i_{[X, \mathbf{h}]} \alpha(Y)$.

\section{REFERENCES}

[1] L. Bates and J. Śniatycki, Nonholonomic reduction, Rep. Math. Phys. 32 (1993), no. 1, 99-115.

[2] E. Binz, M. de León, D. Martín de Diego, and D. Socolescu, Nonholonomic Constraints in Classical Field Theories, Rep. Math. Phys. 49 (2002), 151-166.

[3] E. Binz, J. Śniatycki, and H. Fischer, Geometry of classical fields, North-Holland Mathematics Studies, vol. 154, North-Holland Publishing, Amsterdam, 1988.

[4] A. Bloch, P. Krishnaprasad, J. E. Marsden, and R. Murray, Nonholonomic mechanical systems with symmetry, Arch. Rat. Mech. Anal. 136 (1996), no. 1, 21-99.

[5] F. Cantrijn, M. de León, J. C. Marrero, and D. Martín de Diego, Reduction of nonholonomic mechanical systems with symmetry, Rep. Math. Phys. 42 (1998), no. 1-2, 25-45, Pacific Institute of Mathematical Sciences Workshop on Nonholonomic Constraints in Dynamics (Calgary, AB, 1997).

[6] M. de León, D. Martín de Diego, and A. Santamaría-Merino, Symmetries in classical field theory, Int. J. Geom. Meth. Mod. Phys. 1 (2004), no. 5, 651-710.

[7] M. de León, M. McLean, L. Norris, A. Roca, and M. Salgado, Geometric structures in field theory, preprint, available at math-ph/0208036 (2002).

[8] M. Gotay, J. Isenberg, and J. Marsden, Momentum Maps and Classical Relativistic Fields. Part I: Covariant Field Theory, Preprint, available as physics/9801019.

[9] M. Gotay, J. Isenberg, and J. Marsden, Momentum Maps and Classical Relativistic Fields. Part II: Canonical Analysis of Field Theories., Preprint, available as math-ph/0411032.

[10] I. Kolář, P. Michor, and J. Slovák, Natural operations in differential geometry, Springer-Verlag, Berlin, 1993.

[11] A. Kriegl and P. Michor, The Convenient Setting of Global Analysis, Mathematical Surveys and Monographs, vol. 53, AMS, Providence (RI), 1997.

[12] O. Krupková, Partial differential equations with differential constraints, J. Diff. Eq. 220 (2005), no. 2, 354-395.

[13] A. Santamaría-Merino, Métodos Geométricos en Teorías Clásicas de Campos e Integración Numerica, Ph.D. thesis, Universidad Carlos III de Madrid, 2005.

[14] D. J. Saunders, The Geometry of Jet Bundles, London Mathematical Society Lecture Note Series, vol. 142, Cambridge University Press, 1989.

[15] J. Vankerschaver, F. Cantrijn, M. de León, and D. Martín de Diego, Geometric aspects of nonholonomic field theories, To appear in Rep. Math. Phys. Preprint available as math-ph/0506010.

Joris Vankerschaver: Department of Mathematical Physics and Astronomy, Ghent University, KrijgslaAn 281, B-9000 Ghent, Belgium

E-mail address: Joris.Vankerschaver@UGent.be 